\title{
A Computational Approach to Fractal Analysis of a Cityscape's Skyline
}

\author{
Stephan K. Chalup, ${ }^{1 \dagger}$ Naomi Henderson, ${ }^{1}$ Michael J. Ostwald ${ }^{2}$ and Lukasz Wiklendt ${ }^{1}$ \\ ${ }^{1}$ School of Electrical Engineering and Computer Science, The University of Newcastle, \\ Callaghan 2308, Australia \\ ${ }^{2}$ School of Architecture and Built Environment, The University of Newcastle, Callaghan 2308, \\ Australia \\ ${ }^{\dagger}$ Corresponding author: Tel: 024921 6080; Fax: 024921 6929; Email: \\ stephan.chalup@newcastle.edu.au
}

[Suggested running head: Fractal Analysis of a Cityscape’s Skyline]

\begin{abstract}
This study proposes a semi-automated approach for cityscape analysis which is based on calculating the fractal dimension of a cityscape's skyline. A software tool was developed which consists of an intensity-based skyline extraction module combined with a box-counting approach for calculation of the fractal dimension. Obstacles such as power-lines, vertical poles or cranes which interrupt the skyline can automatically be excluded from the analysis. The paper describes the methods involved and presents three pilot experiments using the new approach which indicate that: (1) If trees intersect the skyline they typically increase its fractal dimension; (2) Different types of cities can be distinguished by their characteristic skylines; (3) The process to determine the best fit skyline in an image can require user intervention. It can be semiautomated by using the local minima of the skyline's fractal dimension which is interpreted as a function of the image's intensity cut-off values.
\end{abstract}

Keywords: Cityscape, Fractal dimension, Image processing, Sky segmentation, Skyline. 


\section{Introduction}

Human well-being is connected to features of the built environment. Although the latter is an intuitively acceptable fact, research into the nature of this relationship has turned out to be a challenging trans-disciplinary undertaking. A recent overview of studies addressing various psychosocial features of urban neighbourhoods that can affect residents is part of a study by Spokane and co-authors (2007). Behavioural survey methods employing human test subjects are naturally dependent on a variety of subject-related factors which can affect an architectural evaluation. It was found, for example, that judgement on landscape preference can depend on the nationality (Purcell, Lamb, Peron, \& Falchero, 1994) or ethnicity (Kaplan \& Talbot, 1988) of test subjects.

The quest for a measure which allows direct comparability and better objectivity has motivated the use of the fractal dimension for architectural image analysis (Mandelbrot, 1983; Bovill, 1996; Ostwald, Vaughan, \& Tucker, 2008). A review of studies on visual perception suggests that people aesthetically prefer patterns of mid-range fractal dimensions (Taylor, 2006). This observation was corroborated by physiological pilot experiments based on skin-conductance measurements which indicated that mid-range fractal dimensions have the most positive effect on subjects' stress (Taylor et al., 2005). A recent investigation used quantitative electroencephalography (qEEG) to record psycho-physiological responses in the cortex of subjects viewing computer-generated fractal silhouettes which underwent controlled changes of their fractal dimension. The results of this study confirmed that mid-range fractal dimensions play a unique role in visual perception (Hagerhall et al., 2008).

Architectural image analysis often focuses on the complexity of house façades and streetscapes. However, for the aesthetic assessment of distant urban views, the complexities of city skylines are at least as important (Heath, Smith, \& Lim, 2000). The skyline is the contour of the sky 
segment in an image and its fractal dimension is regarded as an important feature which may be used to characterise natural scenes (Keller, Crownover, \& Chen, 1987; Keller, Chen, \& Crownover, 1989; Hagerhall, Purcell, \& Taylor, 2004). Psychological eye-tracking experiments have demonstrated that contours with high intensity gradients attract subjects' attention (Rayner \& Pollatsek, 1992). Fractal analysis of urban skylines has previously been conducted by several studies (Cooper, 2000, 2003; Oku, 1990). The theory of contextual fractal fit implies that cityscapes look better if the fractal dimension of their skyline matches the fractal dimension of the environment (Bovill, 1996; Stamps, 2002).

A general aim of the present study is to understand better the impact of skyline complexity on aesthetic judgement and to develop a software tool for architectural image analysis which can help to improve comparability and objectivity. This article contributes a method which allows extraction and fractal analysis of an approximation of the skyline from digital images of cityscapes. The experimental section illustrates how to determine the skyline even in the presence of obstacles such as power-lines, poles and cranes which intersect the skyline.

In order to extract the skyline in digital images, regions which contain sky have to be identified. Specific methods for detection of sky regions have previously been developed for various applications. These include photographic image manipulation and image/video enhancement, as well as areas such as image understanding or semantic image retrieval where the sky region can help to determine the orientation of an image or to identify outdoor images. Luo and Etz (2002) proposed a model-based sky detection method that incorporates colour classification by a multilayer perceptron and a physics-motivated sky signature validation. The sky detection approach of Gallagher, Luo and Hao (2004) used a two-dimensional polynomial model of blue sky in an image to improve the approach of Luo and Etz (2002). The task of real-time sky region segmentation to improve image quality in video image streams was approached by using a 
combination of colour, texture and position information (Herman \& Bellers, 2002; Herman \& Janssen, 2004; Herman \& Bellers, 2005). In a similar series of studies probabilistic models were computed using colour, position and texture features in order to obtain a pixel-precise segmentation of sky regions (Zafarifar \& de With, 2006a,b; Zafarifar \& de With, 2007). These approaches were further developed into real-time implementations (Quach, 2006; Quach et al., 2007).

The application goal of the detection of sky regions in the present study is the extraction of skyline in an image in order to calculate its fractal dimension. In contrast to the work cited above on video-stream processing, our criterion for calculation of the sky region's boundary is not processing speed but precision with respect to the fractal properties of the cityscape's skyline. Objects in the sky or foreground that are not part of the cityscape are to be excluded from the skyline analysis by making them invisible through the segmentation process. This is in contrast to previous work, for example, on sky detection for the purposes of unmanned aerial vehicles avoiding obstacles (McGee, Sengupta, \& Hedrick, 2005; Todorivic \& Nechyba, 2003), where objects in the sky had to be identified as obstacles, at high speed. 


\section{Method}

The basic approach developed in this study can extract the skyline from standard images where the sky is a connected component adjacent to the upper edge of the image and where the sky region is of greater brightness than the rest of the image. Special cases and exceptions require separate treatment and will be addressed in Section 2.2 below.

\section{Basic Sky Segmentation and Skyline Extraction}

The skyline extraction method works on greyscale images with pixel values scaled in the range $[0,1]$. Colour images are converted into greyscale before the method is applied. The method is based on the observation that, in most images, the sky region is brighter than the rest of the image. First the image is filtered by using a $3 \times 3$ pixel block to assign the value

$$
\text { outpixel }=\operatorname{ceil}(a \cdot \operatorname{maxval}-b \cdot \operatorname{stdev}+c \cdot(\text { inpixel }-1))
$$

to the centre pixel of the block, where stdev = standard deviation of the 9-pixel block, maxval = maximum value of the 9-pixel block, inpixel $=$ initial value of the centre pixel. The value of outpixel is clamped to $[0,1]$. The parameters $a, b$, and $c$ were tuned by hand to $a=0.2, b=30$, and $c=0.05$ in a series of pilot tests. The image obtained at this stage of the process is shown in Figure 1(iii).

In the second step of the method the edges of the sky region are extracted. The Matlab function bwboundaries, which traces the exterior boundaries of objects in a binary image, is used to extract the edges of each blob in the filtered image (Matlab, 1992). Edges that touch the top margin of the image are added to the skyline. Finally, pixels that run along the margin of the image are removed leaving only the skyline (second row of Figure 2).

\section{<Insert Figure 2 about here>}




\section{Processing Special Cases}

The sky segment in an image is generally connected to the upper edge of the image, it is typically blue and it is brighter than the rest of the image. However, there are special cases which differ from this standard situation and which require special treatment so that the skyline can be extracted and appropriately analysed.

For example, the first row of Figure 2 shows images containing power-lines, cables, vertical poles or cranes. While these objects are part of the built environment, their presence in images can add irrelevant components to the skyline which would disturb an architectural analysis because the obstacles can alter the results of fractal dimension calculation (see caption of Figure 2). Utilising the unique visual properties of these obstacles we can automatically remove them from the skyline analysis. The removal process, which is described in the following subsections, involves four stages; intensity classification of sky, neighbouring sky calculation, neighbouring texture calculation, and secondary sky classification.

Intensity classification of sky. The basic sky classification method described in Section 2.1 uses a $3 \times 3$ pixel classifier and is fully automated. However, this does not give enough detail in problematic cases where thin obstacles interrupt the skyline, such as wires, cables or thin poles, where an accuracy of 1 pixel is required. An example of such a challenging input image, its greyscale conversion (which could involve additional histogram equalisation) and a skyclassified copy are shown in Figures 1(i), (ii), and (iii), respectively. In order to classify the sky in these special cases the observation is used that sky is typically brighter than buildings and each pixel of the greyscale image is thresholded by an intensity cut-off value (co) where pixel values are assumed to be in the range from 0 to 255. Pixel values above the intensity cut-off are classed as sky, others are classed as non-sky. This gives a sky-classified image accurate to one pixel (sky 
is represented by white and non-sky by black in Figure 1(iv)). The intensity cut-off value can initially be chosen manually using visual inspection of the obtained skylines. We propose to use a semi-automated approach which first calculates the fractal dimension of valid candidate skylines obtained for a series of cut-off values. Our pilot experiments suggest that the cut-off value which corresponds to a local minimum of the fractal dimension most probably determines the locally best skyline within the set of candidates (see Figures 3 and 7 and Section 3).

\section{<Insert Figure 3 about here>}

Neighbouring sky calculation. Some of the problematic areas of images tend to include narrow objects such as power-lines, cables, vertical poles or cranes which have neighbouring regions of sky. To detect these, a neighbouring sky calculation is performed. For each pixel the sum of neighbouring sky pixels is calculated for a $7 \times 7$ block surrounding the pixel. Consequently the sum of the sky values for each $7 \times 7$ block ranges from 0 to 49 , where 0 indicates that there are no other pixels classified as sky and a value of 49 indicates that the centre pixel itself and all surrounding pixels are classified as sky.

Neighbouring texture calculation. A visual property that differentiates sky from non-sky is the texture of the area. Within sky regions, even if they include clouds, there are smooth transitions of intensity values. If there are regions in the image that contain buildings then there are typically sharp transitions of intensity values. These transitions can be measured using the image processing technique of edge detection.

The present system employs standard Sobel edge detection with a threshold value of 0.03 to search the image for edges (Matlab, 1992). Input to the edge detection function is a greyscale image. The return is 1 for any point where the gradient of intensity change is above the threshold value and 0 otherwise (where $1=$ white and $0=$ black in Figure $1(v)$ ). 
For each pixel of the image, the sum of neighbouring edge pixels was calculated for a $7 \times 7$ region surrounding the pixel. The sum of the edge values ranges from 0 to 49, where 0 indicates that there are no edge values surrounding the pixel and 49 indicates that the pixel itself and all neighbouring pixels are edges (which is unlikely).

Secondary sky classification. The neighbouring sky and texture calculations can be used as additional information when classifying a pixel. The presence of a significant amount of sky surrounding the pixel suggests that it too should be considered as part of the sky. Furthermore a lack of surrounding edges suggests that the pixel is not part of the buildings being analysed.

The secondary sky classification process uses the intensity-classified image and re-classifies it based on the additional neighbouring sky and texture calculations. For any pixel classified as not sky it calculates its significance based on neighbouring sky and texture information. This is done using the following logical expression:

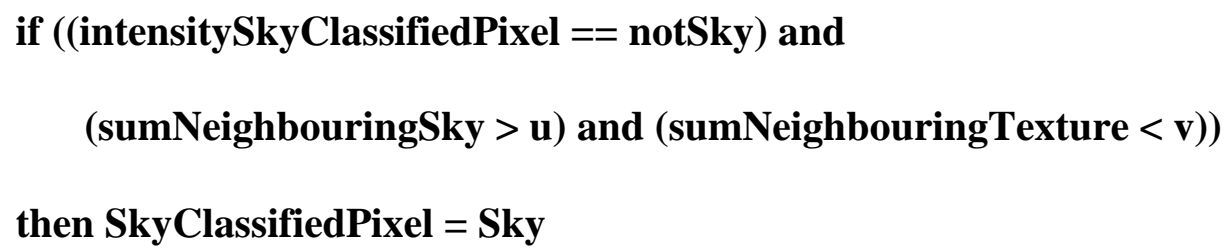

The values for $\mathbf{u}$ and $\mathbf{v}$ were tuned using several calibration tests on a set of example images: $\mathbf{u}$ was set to 10 and $\mathbf{v}$ to 30 . This means that a non-sky classified pixel with more than 10 pixels of sky and less than 30 pixels of edges neighbouring it is not included in the skyline analysis. The resulting filtered image can be seen in Figure 1(vi).

The skyline extraction function is then applied to the secondary sky classified image. Examples of images where power lines, poles and cranes have been removed are shown in the third row of Figure 2. 


\section{Fractal dimension calculation by box-counting}

Fractal dimension calculation by box-counting for architectural image analysis was applied by hand, for example, in the work of Bovill (1996). Automated versions of box-counting were employed in recent computer-assisted studies (Foroutan-Pour, Dutilleul, \& Smith, 1999; Ostwald \& Tucker, 2007; Ostwald et al., 2008). To avoid any issues with scaling, all images in the present study had equal size. Input to the box-counting function was a black-and-white image of the extracted skyline. The iterative function creates at each step a grid which has half the number of boxes in each dimension (i.e., a quarter of the total number of boxes) than the grid in the previous step. At each step the function coarsens the grid by combining each $2 x 2$ subgrid of boxes into a single box, and labels the box as 1=grey if there was at least one grey box in the 2x2 subgrid (see Figure 4) and otherwise the new box is labelled $0=$ white. Two vectors, $r$ and $N$, are created.

\section{$<$ Insert Figure 4 about here $>$}

The i-th component of $r$ is the reduction factor $r_{i}=2^{(i+1)}$ at iteration step i. The i-th component of $\mathrm{N}$ is the number of grey boxes in the scaled grid at step i. A line is fitted through the points $\left(\log \left(1 / \mathrm{r}_{\mathrm{i}}\right), \log \left(\mathrm{N}_{\mathrm{i}}\right)\right)$, where the first and last elements in $\mathrm{N}$ and $\mathrm{r}$ are suppressed for a better line fit. The fractal dimension is obtained as the slope of that line.

\section{Experiments}

The method was implemented in Matlab (Matlab, 1992) and three hypotheses were investigated in pilot experiments:

Hypothesis I: Trees as part of the skyline increase its fractal dimension. 


\section{<Insert Figure 5 about here>}

Photographs of the same streetscape, with trees and without trees, as shown in the example in Figure 5, were analysed. In all cases the images with trees showed a higher fractal dimension, which is a plausible result given that trees typically have a higher fractal dimension than houses.

\section{<Insert Figure 6 about here>}

Hypothesis II: The fractal dimension of the skyline is a sensitive measure which can be used to distinguish different types of cityscapes.

The method was applied to images taken at three different locations (representative samples are shown in Figure 6): (1) Amsterdam: The extracted skylines seemed to have a relatively high fractal dimension. The hypothesis is that the mostly historical houses in the images display highly structured shapes and contours. However, closer inspection of the photographs also revealed that several trees intersect the skylines. (2) Sydney: Sydney’s skylines display a medium-range fractal dimension which could be due to the mixture of architectural styles present in the city. (3.) Suzhou: The industrial area of Suzhou is characterised by straight and modern apartment and office buildings. The extracted skylines in our experiments had a relatively low fractal dimension. These pilot experiments, which only involved a small number of samples, suggest that the method has the potential to distinguish different types of cityscapes provided a sufficiently large image database is used.

Hypothesis III: Local minima of the fractal dimension can be used to determine the intensity cutoff value which controls the position of the skyline. 
The intensity cut-off value can be used to control the skyline approximation (see Section 2.2 and Figures 3 and 7). The lines with markers (o), $(*)$, and (x) in Figure 7 correspond to the images in columns 1, 2, and 3, respectively, of Figure 3. The best fit skylines were determined for cut-off values 210, 170, and 190 for the lines with markers (o), (*), and (x), respectively. Each of these points corresponds to a local minimum of the fractal dimension (highlighted by arrows in Figure 7) which means this method determines locally the least fractal or smoothest skyline. The line with marker $(*)$ in Figure 7 is an example of where the selected local minimum (arrow at $\mathrm{co}=170$ ) is not the global minimum. The selected local minimum corresponds to the skyline in the image in row 2 and column 2 of Figure 3, while the global minimum appears to be at co=190 and corresponds to the skyline in the image in row 3 and column 2 of Figure 3. The latter had to be rejected by the user because the skyline jumps some gaps between buildings. In summary, these experiments suggest that qualitative features of the skyline such as "skyline invades façades”, "skyline approximates top of buildings”, or "skyline takes off into the sky region" appear to be locally stable. Therefore the local selection of the skyline can be automated by using local minima of the fractal dimension. However, globally the process is only semi-automated and requires user input whenever high level context-dependent decisions are necessary to select the most appropriate local minimum.

\section{Conclusion}

An important motivation for this study is the appearance of recent physiological studies which use skin-conductance measurements or qEEG and indicate that the fractal dimension is an important feature for architectural image analysis. The present study presented details of a new approach for semi-automated fractal analysis of a cityscape's skyline which can eliminate 
obstacles such as power-lines, cranes and poles.

Pilot experiments showed that calculating a sensible skyline approximation can be an unstable process which depends on various characteristics of the input image. We proposed a new method to control the skyline approximation locally via intensity cut-off values where the best approximation is determined by a suitable local minimum of the corresponding fractal dimension.

In our experiments trees typically increased the skyline's fractal dimension. Future investigation may address the question whether it is an advantage to remove trees or how to include them in the fractal analysis process. Other topics for future research include extreme lighting conditions, distinctive cloud structure, extreme weather conditions, and other non-standard situations.

This interdisciplinary study of architecture and computer science demonstrates how architectural analysis of cityscapes critically depends on subtle parameters in image processing. Researchers in architectural image analysis may find the presented method useful when investigating images of cityscapes where a precise analysis of the skyline is required.

\section{Acknowledgements}

This project was supported by ARC discovery grant DP0770106, “Shaping social and cultural spaces: the application of computer visualisation and machine learning techniques to the design of architectural and urban spaces”. 


\section{References}

Bovill, C. (1996). Fractal Geometry in Architecture and Design. Design Science Collection. Boston: Birkhäuser.

Cooper, J. C. (2000). The Potential of Chaos and Fractal Analysis in Urban Design. Oxford: Joint Centre for Urban Design, Oxford Brookes University.

Cooper, J. C. (2003). Fractal assessment of streetlevel skylines - a possible means of assessing and comparing character. Urban Morphology, 7, 73-82.

Foroutan-Pour, K., Dutilleul, P., \& Smith, D. L. (1999). Advances in the implementation of the box-counting method of fractal dimension estimation. Applied Mathematics and Computation 105(2-3), 195-210.

Gallagher, A. C., Luo, J., \& Hao, W. (2004). Improved blue sky detection using polynomial model fit. In: 2004 International Conference on Image Processing, 2004. ICIP '04. Vol. 4. IEEE, pp. 2367-2370.

Hagerhall, C. M., Purcell, T., \& Taylor, R. P. (2004). Fractal dimension of landscape silhouette as a predictor of landscape preference. The Journal of Environmental Psychology, 24, 247-255.

Hagerhall, C. M., Laike, T., Taylor, R. P., Küller, M., Küller, R., \& Martin, T. P. (2008). Investigations of human EEG response to viewing fractal patterns. Perception, 37(10), 1488 1494.

Heath, T., Smith, H. G. G., \& Lim, B. (2000). Tall buildings and the urban skyline: The effect of visual complexity on preferences. Environment and Behavior, 32, 541-556.

Herman, S., \& Bellers, E. (2002). Locally-adaptive processing of television images based on realtime image segmentation. In: International Conference on Consumer Electronics, 2002. ICCE. IEEE, pp. 66-67.

Herman, S., \& Bellers, E. (2005). Adaptive segmentation of television images. European Patent 


\section{EP 1573673.}

Herman, S., \& Janssen, J. (2004). System and method for performing segmentation-based enhancements of a video image. European Patent EP 1374563.

Kaplan, R., \& Talbot, J.F. (1988). Ethnicity and preference for natural settings: A review and recent findings. Landscape Urban Planning, 15, 107-117.

Keller, J. M., Chen, S., \& Crownover, R. M. (1989). Texture description and segmentation through fractal geometry. Computer Vision, Graphics, and Image Processing, 45, 150-166.

Keller, J. M., Crownover, R. M., \& Chen, R. Y. (1987). Characteristics of natural scenes related to the fractal dimension. IEEE Transactions on Pattern Analysis and Machine Intelligence, 9, $621-627$.

Luo, J., \& Etz, S. (2002). A physical model-based approach to sky detection in photographic images. IEEE Transactions on Image Processing, 11(3), 201-212.

Mandelbrot, B. B. (1983). The Fractal Geometry of Nature. New York: W. H. Freeman.

Matlab (1992). User’s Guide. URL http://www.mathworks.com/, (retrieval date 10.10.2008).

McGee, T. G., Sengupta, R., \& Hedrick, J. K. (2005). Obstacle detection for small autonomous aircraft using sky segmentation. In: Proceedings of the IEEE International Conference on Robotics \& Automation, Barcelona, Spain.

Oku, T. (1990). On visual complexity of the urban skyline. Journal of Planning, Architecture and Environmental Engineering, 412, 61-71.

Ostwald, M. J., \& Tucker, C. (2007). Reconsidering Bovill's method for determining the fractal geometry of architecture. In: J. Coulson, D. Schwede, \& R.Tucker (Eds.). Towards solutions for a liveable future: Progress, Practice, Performance, People. Proceedings of the 41st Annual Conference of the Architectural Science Association (ANZAScA), Melbourne (pp. 182-190).

Ostwald, M. J., Vaughan, J., \& Tucker, C. (2008). Characteristic visual complexity: Fractal dimensions in the architecture of Frank Lloyd Wright and Le Corbusier. In: K. Williams (Ed.), 
Nexus: Architecture and Mathematics (pp. 217-232). Turin: K. W. Books and Birkhäuser.

Purcell, A. T., Lamb, R. J., Peron, E. M., \& Falchero, S. (1994). Preference or preferences for landscape? Journal of Environmental Psychology 14(33), 195-209.

Quach, N. T. (2006). Real-time sky-detection implementation. MSc thesis, Computer Engineering, Department of Electrical Engineering, Faculty of Electrical Engineering, Mathematics and Computer Science, Delft University of Technology, Mekelweg 4, 2628 CD Delft, The Netherlands.

Quach, N. T., Zafarifar, B., \& Gaydadjiev, G. N. (2007). Real-time FPGA-implementation for blue-sky detection. In: IEEE International Conference on Application-Specific Systems, Architectures and Processors, ASAP 2007, Montreal, Quebec, Canada, July 8-11, 2007. (pp. 7682) IEEE Computer Society.

Rayner, K. \& Pollatsek, A. (1992). Eye movements and scene perception. Canadian Journal of Psychology. 46, 342-376.

Spokane, A. R., Lombard, J. L., Martinez, F., Mason, C. A., Gorman-Smith, D., Plater-Zyberk, E., Brown, S. C., Perrino, T. \& Szapocznik, J. (2008). Identifying streetscape features significant to well-being. Architectural Science Review, 50(3), 234-245.

Stamps, A. E. (2002). Fractals, skylines, nature and beauty. Landscape and Urban Planning, 60(3), 163-184.

Taylor, R. P. (2006). Reduction of physiological stress using fractal art and architecture. Leonardo, 39(3), 25-251.

Taylor, R. P., Spehar, B., Wise, J. A., Clifford, C. W.G., Newell, B. R., Hagerhall, C. M., Purcell, T., \& Martin, T. P. (2005). Perceptual and physiological responses to the visual complexity of fractal patterns. Journal of Nonlinear Dynamics, Psychology, and Life Sciences, 9, 89-114. Todorivic, S., Nechyba, M., \& Ifju, P.G. (2003). Sky/ground modelling for autonomous MAV flight. In: Proceedings of the IEEE International Conference on Robotics and Automation 
(ICRA), Volume 1, (pp. 1422-1427), IEEE.

Zafarifar, B., \& de With, P. H. N. (2006a). Adaptive modeling of sky for video processing and coding applications. In: 27th Symposium on Information Theory in the Benelux, June 2006, Noordwijk, The Netherlands. (pp. 31-38), WIC (Werkgemeenschap for Informatie- en Communicatietheorie).

Zafarifar, B., \& de With, P. H. N. (2006b). Blue sky detection for picture quality enhancement. In: J. Blanc-Talon, W. Philips, D. Popescu,, \& P. Scheunders (Eds.), Advanced Concepts for Intelligent Vision Systems, 18th International Conference, ACIVS 2006, Antwerp, Belgium, September 18-21, 2006, Proceedings. (pp. 522-532). Vol. 4179 of Lecture Notes in Computer Science. Springer: Berlin / Heidelberg.

Zafarifar, B., \& de With, P. H. N. (2007). Blue sky detection for content-based television picture quality enhancement. In: International Conference on Consumer Electronics, ICCE 2007. Digest of Technical Papers. (pp. 1-2), IEEE. 


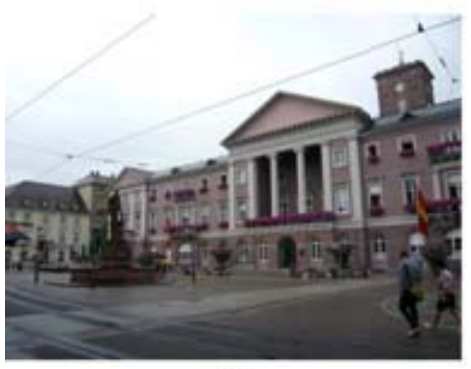

(i)

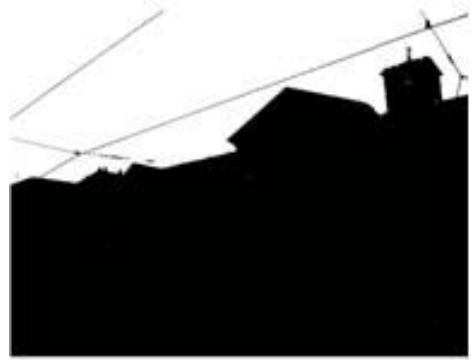

(iv)

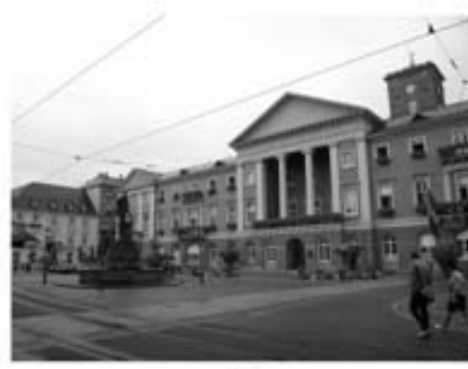

(ii)

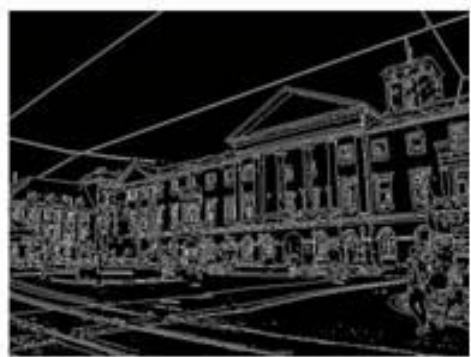

(v)

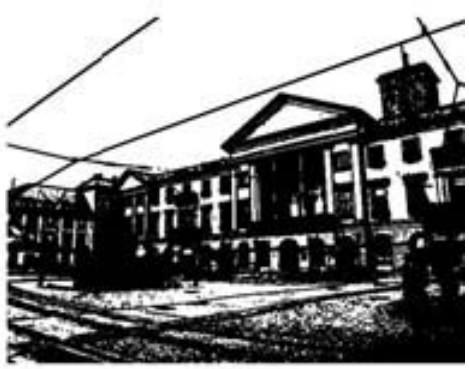

(iii)

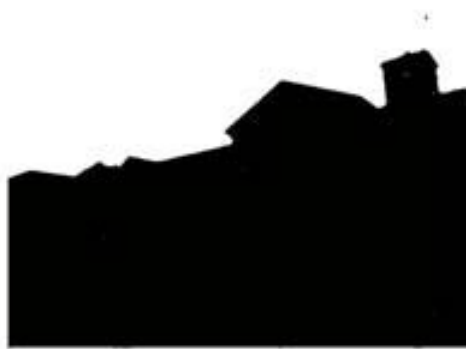

(vi)

Figure 1: Stages of the procedure for processing special cases: (i) Input image (can be in colour); (ii) Greyscale (intensity) image (can include histogram equalization); (iii) Sky classified image using $3 \times 3$ pixel classifier; (iv) Intensity sky classified image using cut-off value = 200; (v) Edge image; (vi) Resulting secondary sky classified image. 

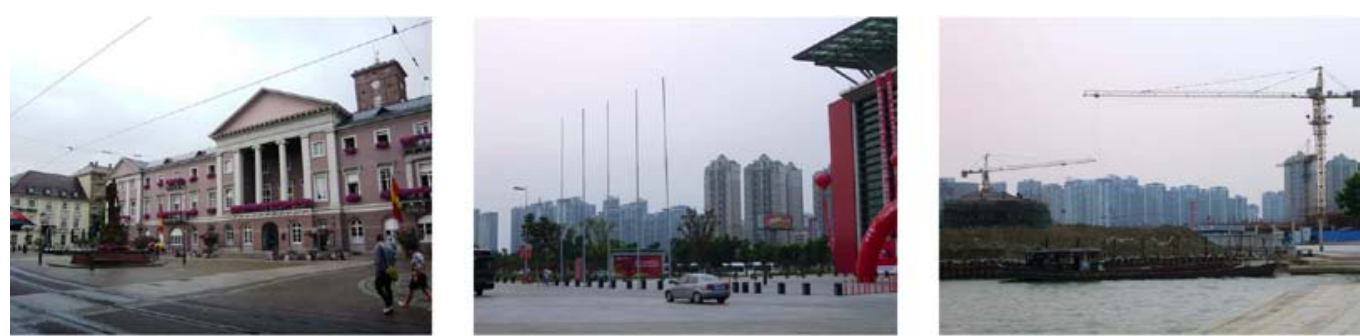

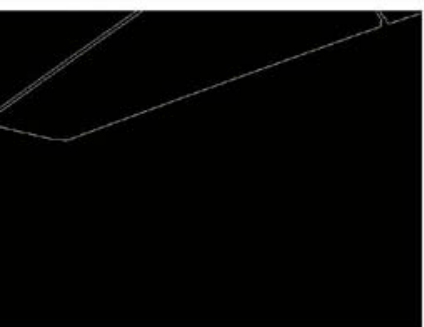

$\mathrm{fd}=1.1392$

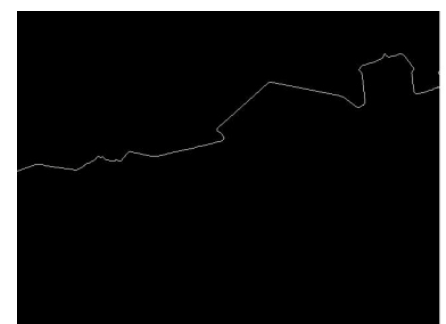

fd=1.0439 (cut-off=210)

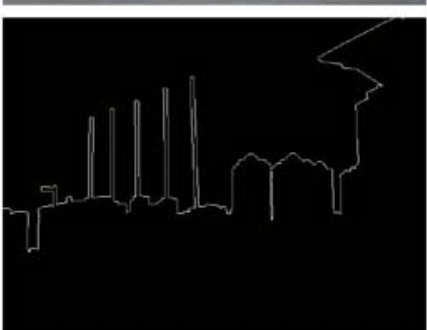

$\mathrm{fd}=1.2234$

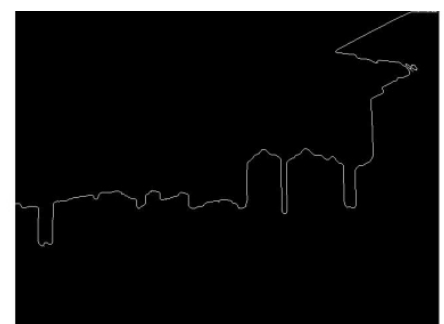

$\mathrm{fd}=1.0999$ (cut-off=170)

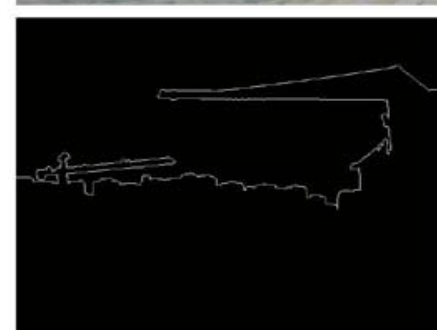

$\mathrm{fd}=1.1381$

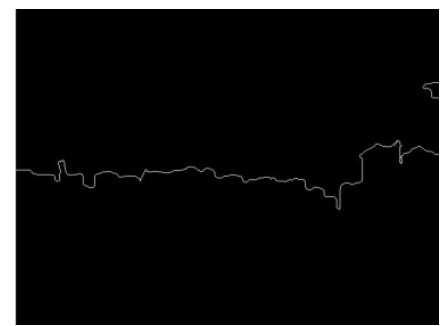

$\mathrm{fd}=0.9297$ (cut-off $=190)$

Figure 2: Skyline extraction and fractal dimension (fd) calculation applied to images containing cables, poles, or cranes. In our tests the fractal dimension of the images without skyline correction (second row) was typically higher than the fractal dimension of the corrected skylines (last row). 


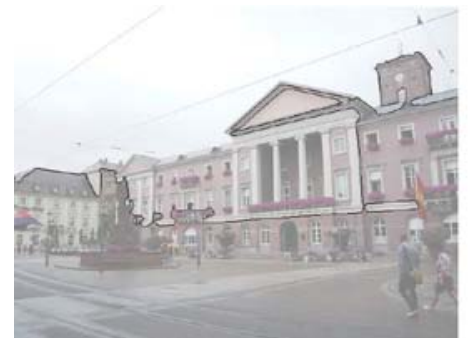

$c o=120: \quad \mathrm{fd}=1.1474$

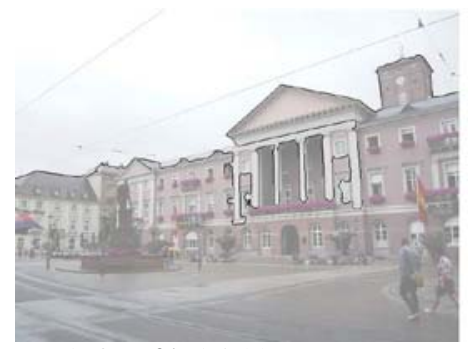

$\mathrm{Co}=170: \quad \mathrm{fd}=1.2559$

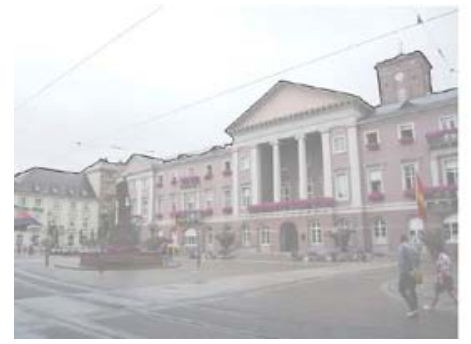

co=190: $\mathrm{fd}=1.0557$

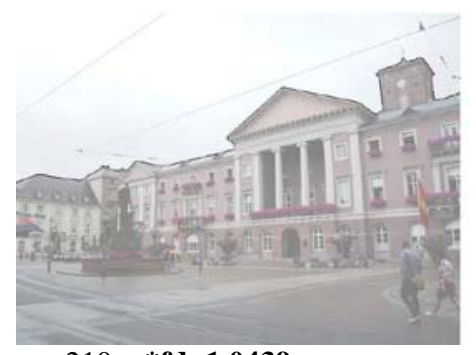

$C o=210: \quad * \mathbf{f d}=\mathbf{1 . 0 4 3 9}$

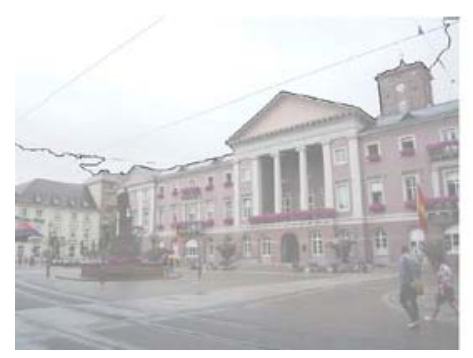

$\mathrm{co}=220: \quad \mathrm{fd}=1.0804$

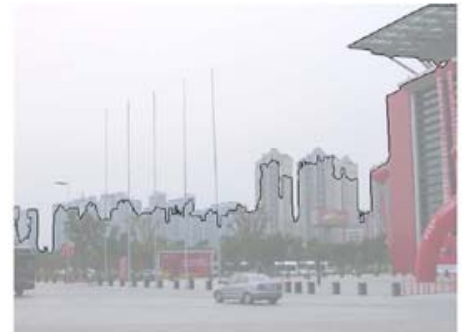

$\mathrm{fd}=1.1735$

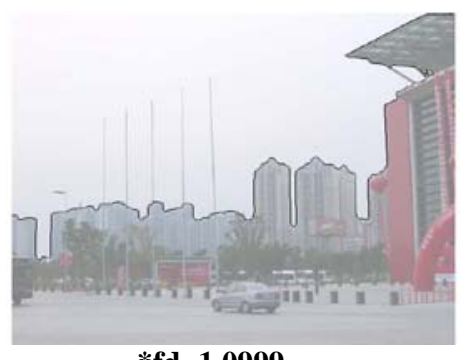

*fd=1.0999

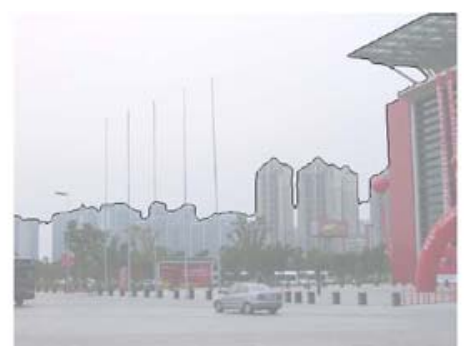

$\mathrm{fd}=1.0582$

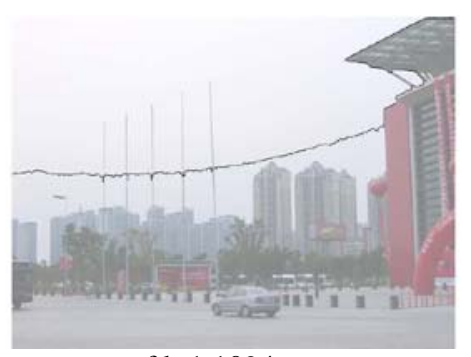

$\mathrm{fd}=1.1094$

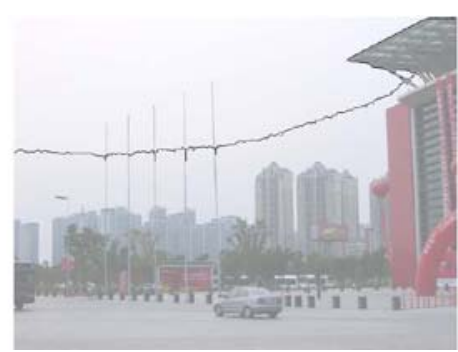

$\mathrm{fd}=1.1271$

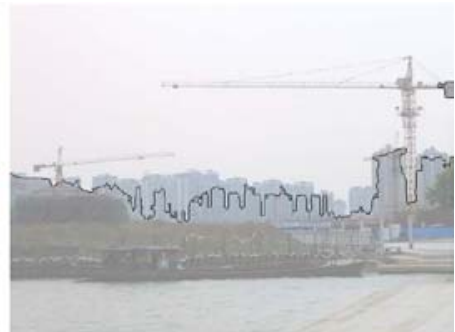

$\mathrm{fd}=1.1626$

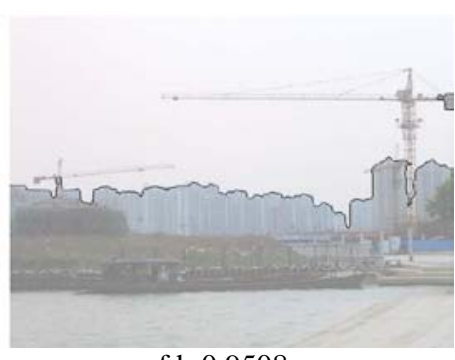

$\mathrm{fd}=0.9508$

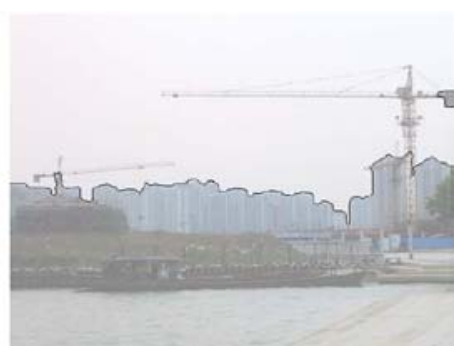

$* \mathbf{f d}=\mathbf{0 . 9 2 9 7}$

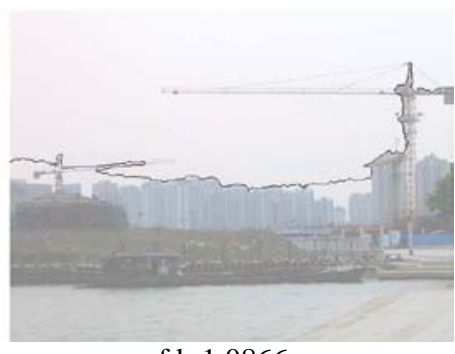

$\mathrm{fd}=1.0866$

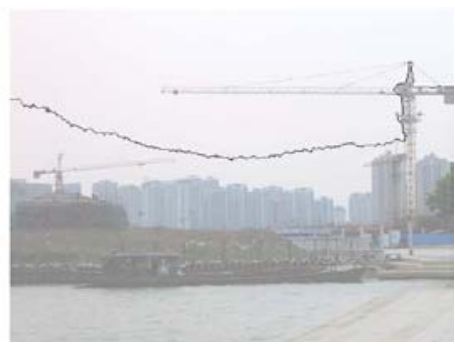

$\mathrm{fd}=1.0783$

Figure 3: Skyline extraction and fractal dimension ( $f d$ ) calculation depending on different intensity cut-off values: co $=120,170,190,210,220$. The best fit skylines (labelled by *fd) were obtained close to cut-off values which generate local minima for the fractal dimension. 
8

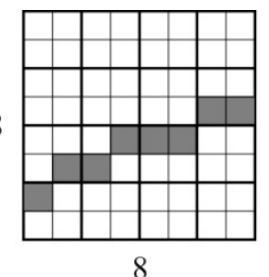

2

reduction

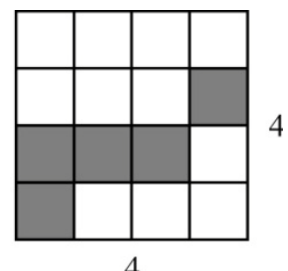

4

Figure 4: Grid reduction step with box size increase in box-counting. 


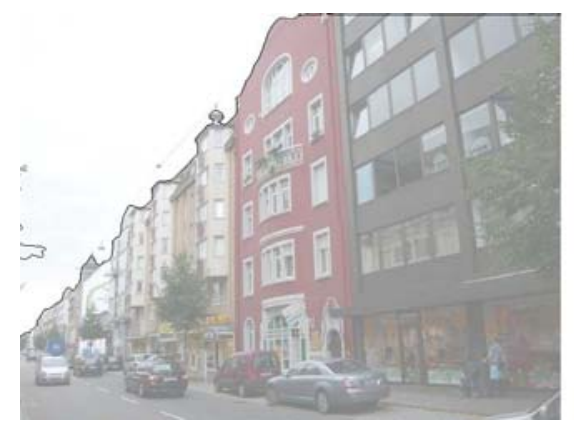

cut-off $=235, \mathrm{fd}=1.0543$

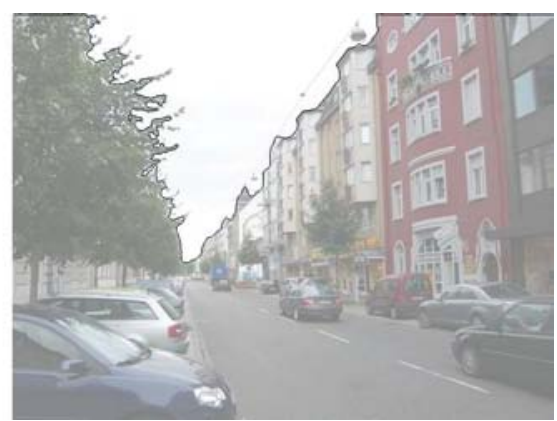

cut-off $=235, \mathrm{fd}=1.1567$

Figure 5: Example of a streetscape without trees (left) and with trees (right). 

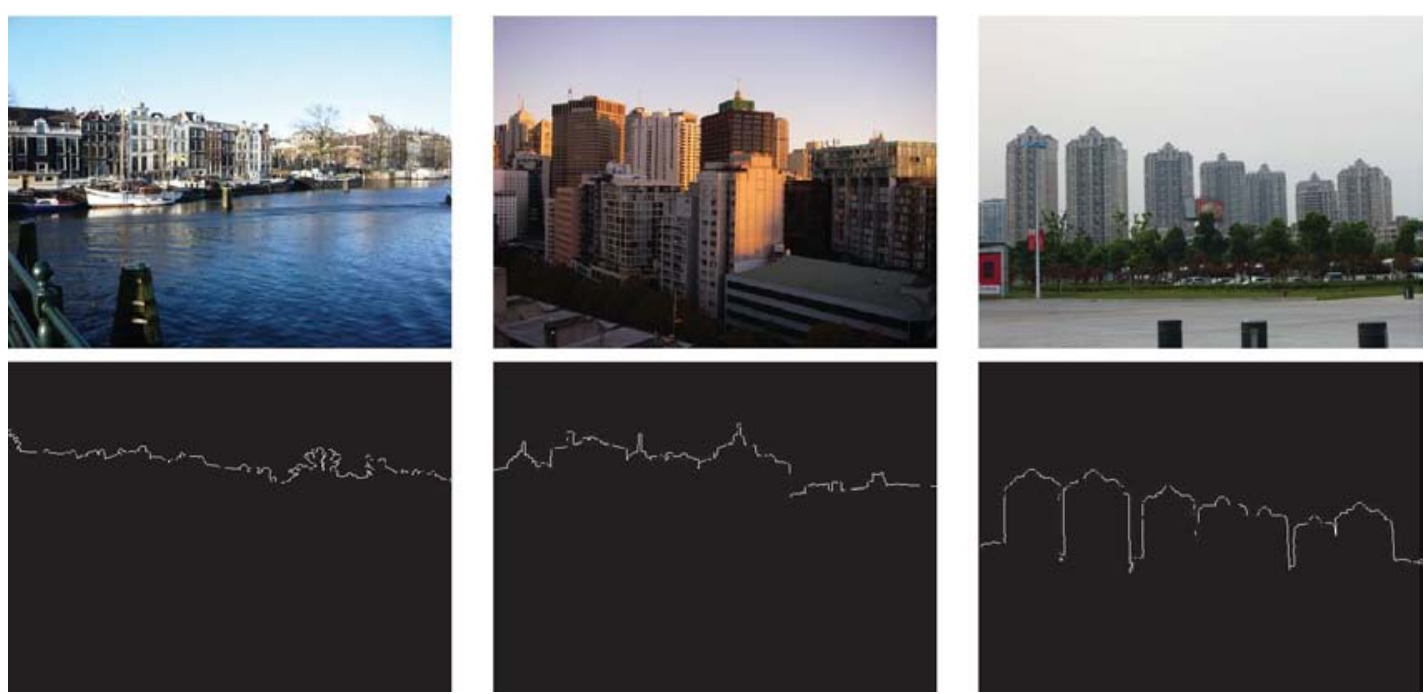

$\mathrm{fd}=1.1229$

$\mathrm{fd}=1.0939$

Amsterdam

Sydney

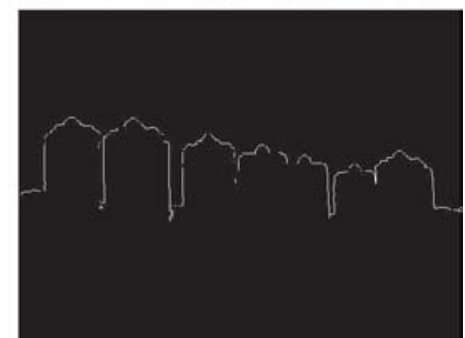

$\mathrm{fd}=1.0591$

Suzhou

Figure 6: Pilot results of the method applied to sample images indicate that skylines of historic cityscapes such as the image of Amsterdam on the left have a higher fractal dimension than, e.g., the skyline of the modern area of Suzhou in the image on the right. 


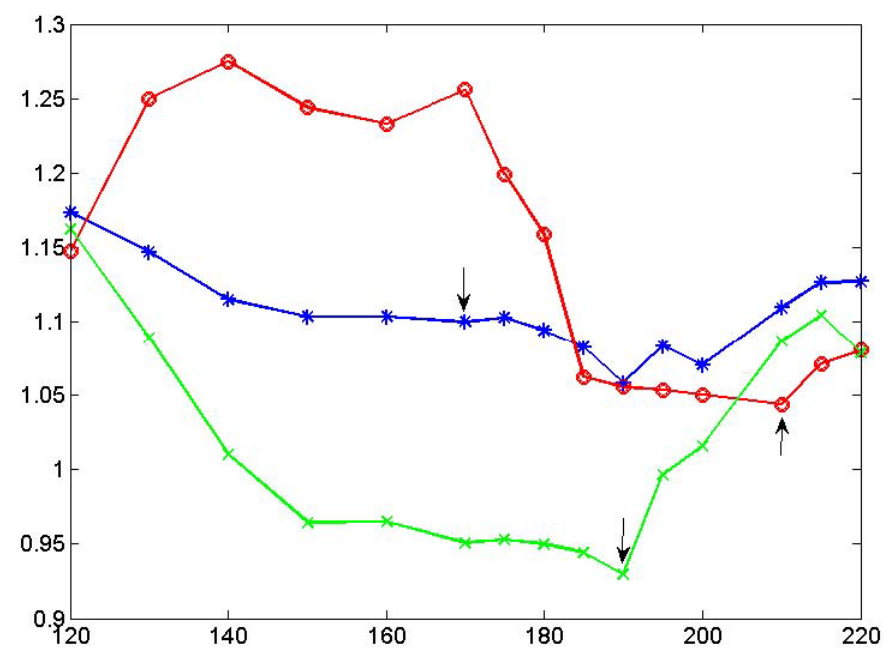

Figure 7: Plot of fractal dimension (vertical axis) versus pixel intensity cut-off values (horizontal axis). Local minima which were used to select skylines of best fit are highlighted by arrows. Lines with markers $(o),\left(^{*}\right)$, and $(x)$ correspond to the images in column 1,2 , and 3 , respectively, in Figure 3. 